DOI: $10.51480 / 1899-5101.14 .1(28) .14$

\title{
CHRISTIAN HOFMANN AND MONICA KIRNER-LUDWIG (EDS.) (2020). TELECINEMATIC STYLISTICS. LONDON: BLOOMSBURY ACADEMIC, 352 PP., ISBN: 9781350042872.
}

This multi-authored collection of articles is a highly valuable and useful contribution to the growing field of research on tele-cinematic stylistics, developing extensively since the early years of the 21st Century. The book revolves around the five major topics of the telecinematic studies: the particular stylistics of genres, sub-genres and formats, both fiction and non-fiction; the specific aspects of various genres and formats (as dialogue, characters, uses of sound or editing etc.); linguistic methodologies, usable and useful in the analysis of film and television; multimodal analysis of film and television texts; representation studies as applied to these texts with their cultural context-relatedness and ideological meanings in view.

Four chapters are, therefore, devoted to the role and functions of the language and the language patterns developed in the telecinematic discourse. The diversified source material varies from feature films, through the soap operas, to sitcoms. The main methodology used here is a corpus analysis, although the scope and specificity of research vary - between the micro-analyses, focused on one particular trait of the telecinematic discourse, to the wide-reaching analysis of the entire corpus of the verbal language of a particular TV station (in this case, Sydney TV) or genre (soap opera).

The second cluster, consisting of five articles, deals with the cinematic discourse, composed of images and other (non-linguistic) resources. The analyzed materials are documentaries, horror movies, comedy/drama, and cinematic trailers. Pragma-stylistics, critical discourse analysis, conversation analysis, close textual reading, and multimodal methodologies are applied here, while the authors combine them to come up with their own qualitative methodologies. Such as, the first creative combination of the analysis of linguistic pragmatic markers and the qualitative analysis of the relationships between characters and mise-en-scene.

The third part - four papers - deals with the intermediality and transcoding or transmodal adaptation, i.e., the transfer of meaning between different semiotic resources. The authors also tackle, albeit not extensively, the issues of intertextuality. They compare and analyze the use of various semiotic modes in the texts transferred from one medium to another (form example from a literary text to a film) or between different layers of the same text (like spoken dialogue vs. captions). The source material here would be historical drama, advertisements, 
and various motion pictures, including Steven Spielberg's adaptation of Tintin comic strips and some Hitchcock films. The methodology draws on pragmatics, and intertextual and semiological tools.

All the authors draw from the shared basic definition of the telecinematic discourse, which in turn is based on the classic definition that was coined in the book "Telecinematic discourse: Approaches to the language of films and television series" by Piazza, Bednarek and Rossi and published in 2011. Still, the editors of "Telecinematic Stylistics", Christian Hofmann and Monica KirnerLudwig propose to broaden the meaning of televisual language to include all kinds of modes and cross-modal links (while also admitting that the visual and aural experiences of the viewer constitute two possibly separate realms). Hofmann and Kirner-Ludwig also - with media convergence in mind - propound the application of the concept of the telecinematic discourse to various media genres. They do not want to restrict the discourse to just fictional genres, nor to only cinematic, even though they also acknowledge the multi-layeredness of the use of language in the film (i.e., not just dialogues, but also monologues, off-screen voice, voice-over, written language, combined with the diegetic and no-diegetic sounds, music etc.).

The editors provide a relatively comprehensive overview of the relevant telecinematic analyses conducted heretofore. The overview helps to place the analyses proposed here in the larger scholarly context. A valuable and interesting part of the book is also the discussion (together with some solutions proposed) of the technical and methodological problems associated with the analysis of audiovisual text, like sources, transcription, the multi-levelled character of communication, designed audiences vs. actual. The authors solve these problems in various ways, while using the existing corpora of media language, their own transcriptions, or the transcriptions found online.

Overall, the publication shows the wide array of tools and analytical devices useful in the analysis of the televisual and cinematic text, thus presenting the cinematic stylistics as the all-encompassing field of analysis of the audiovisual text or discourse in film and television. The object of the analysis of this particular set of tools can be stylistics of televisual and cinematic texts as a whole, particular stylistics of different media genres, formats, particular films or series, or stylistics of chosen directors or directors of photography The methodology developed within its area, though, can be also used for a multitude of other purposes in many research projects - which can be a very useful outcome of this book for scholars using various languages and analyzing a variety of media texts and discourses.

While the collection is undoubtedly valuable and useful, particularly to those interested in media-study methodologies, there are also some shortcomings. Probably most relevant stems from the overarching interest with the spoken 
language. While the authors admit the multimodal nature of the telecinematic text, they either limit their efforts to the analysis of the linguistic layer of the material or only acknowledge its double-layered (language and image) character. The book could probably contribute to the progress of the multimodal analysis of the telecinematic stylistics, but the actual analyses do not practically account for the complex nature of this phenomenon. Another shortcoming will concern mostly non-English speaking readers, and researchers interested in non-English language material. Linguistic analyses, both quantitative and qualitative, are carried out on the English corpora and English texts, respectively. Due to the grammar, syntax and semantic differences between languages, part of them (particularly the most detailed ones, like the research on the demonstrative pronouns in film or on "problem talk" in soap operas) are rather difficult or even impossible to extrapolate. The results are then rather ideographic than universal, and their relevance - for non-English speaking researchers - is thus limited (unless they are used for comparative purposes). This reservation, though does not apply to all the articles. The level of universality is diversified, and some findings (like the interesting categorization of humorous devices in TV series, the application of cooperation principles to the analysis of horror movies, or the attempt to grasp the image-language relationships in the close-captioned films) can easily transcend language limitations.

Despite these limitations, the collection of the essays on telecinematic stylistics is a valuable resource of methodology, interesting insights, and creative solutions for methodological problems. The book can be recommended to both students of media and communication, as well as seasoned media scholars.

Małgorzata Lisowska-Magdziarz JAGIELLONIAN UNIVERSITY, POLAND 